\title{
Avance en el mejoramiento genetico del trigo en Mexico
}

\author{
Arturo HERNÁNDEZ SIERRA \\ Invest. Prog. de Trigo INIFAP-SARH. 4.22.00, Chapingo CP 56230, MÉXICO Mexique
}

Durante el periodo invierno-primavera de $1982-83$ y de 1983-84, se evaluaron 81 variedades de trigo (Triticum aestivum L.) en Roque, Guanajuato y durante 1983-84 y 1984-85 en Chapingo, México. El diseño experimental usado en cada caso fue un látice simple con 2 repeticiones.

El propósito de esta evaluación fue determinar, bajo condiciones controladas de encamado, plagas y royas, el avance genético logrado en el rendimiento de grano y la relación de éste con otros caracteres de planta de las variedades mexicanas de trigo. El avance se midió comparando el rendimiento promedio de las variedades más representativas de cada época, con la variedad introducida y usada en siembras comerciales durante el período 1948-1951, « Supremo 211 ».

Los resultados indican que aunque el rendimiento se estabilizó durante los períodos de 1966-70 y 1976-80, el potencial de rendimiento de las variedades liberadas sucesivamente desde 1950 hasta 1982 , ha sido incrementado en $74 \%$. Esto representa una tasa promedio de ganancia de grano de $60 \mathrm{~kg}$ ha ${ }^{1}$ año '.

Dado que la investigación sobre trigo en México se inició formalmente en 1945, esto significa que en los 37 años de investigación en este cultivo el rendimiento se ha incrementado en $2 \%$ por año. Este aumento en el rendimiento de las variedades nuevas, se atribuye a una mayor resistencia al encamado y a las enfermedades, y a una capacidad mayor para producir grano por unidad de tiempo y de superficie.

Palabras clave : Tricicum aestivum L., programa mexicano de trigo, rendimiento trigo en Mexico, componentes de rendimiento, tasa de llenado de grano.

\section{SUMMARY}

Progress from wheat breeding in Mexico.

During the winter-spring of 1982-83 and 1983-84, 81 wheat varieties (Triticum aestivum L.) were evaluated in Roque, Guanajuato and during 1983-84 and 1984-85 in Chapingo, Mexico. The experimental design used in each case was a simple lattice with 2 replications. The purpose of this evaluation was to determine, under conditions of controlled lodging, pest and disease, the progress that has been achieved in grain yield, and the relationship of this with other plant characteristics of the Mexican wheat varieties. Progress was measured comparing the average yield of the more representative varieties of each period with Supremo 211, an introduced variety that was commercially grown during the period 1948-51. The results indicate that although yield reached a plateau during the periods of 1966-70 and 1976-80, the grain yield potential of the varieties successively released from 1950 until 1982 has increased by $74 \%$, for an average annual rate of gain in grain of $60 \mathrm{~kg} \mathrm{ha}^{-1} \mathrm{yr}^{-1}$.

Given that the Mexican wheat breading program formally started in 1945, it means that in the 37 years of research in this crop, the yield potential has increased $2 \%$ per year. This increase in the yield of the new varieties can be attributed to better lodging and disease resistance, and to a greater grain yield capacity per unit area and time.

Additional key words : Triticum aestivum, Mexican wheat program, wheat yield in Mexico, yield components, grain filling rate.
Le progrès génétique obtenu pour le blé au Mexique.

Pendant la période hiver-printemps 1982-1983 et 1983-1984, on a évalué 81 variétés de blé (Triticum aestivum L.) à Roque, Guanajuato, et en 1983-84 et 1984-85 à Chapingo, México, avec un modèle de Latice simple avec deux répétitions.

L'objet de ce travail a été de déterminer, sous contrôle de la verse, des insectes nuisibles et de la rouille, le progrès génétique obtenu en rendement en grain et son rapport avec d'autres propriétés de la plantes, ceci pour des variétés mexicaines de blé. La progression obtenue par l'amélioration génétique a été mesurée par la comparaison du rendement moyen des variétés les plus représentatives de chaque époque, avec la variété introduite que l'on utilisait pendant la période 1948-1951 (Supremo 211).

Bien que les résultats montrent une stagnation du rendement pendant les périodes de 1966-70 et 1976-80, le potentiel de rendement des variétés obtenues depuis 1950 jusqu'en 1982 s'est accru de $74 \%$, Ce qui représente un taux moyen d'augmentation de 60 kilogrammes de grain par hectare par an.

Si l'on situe en 1945 le commencement de la recherche sur le blé au Mexique, pour 37 ans de recherche (1945-1982) la progression en rendement de blé a été de $2 \%$ par an. Cette augmentation du rendement des nouvelles variétés, 
est attribuée à une forte résistance à la verse et aux maladies, et à une capacité accrue des variétés pour la production de grain par unité de temps et de surface.

Mots clés additionnels : Triticum aestivum L., recherche sur le blé au Mexique, composantes du rendement, taux de remplissage du grain.

\section{INTRODUCCION}

Antes de iniciar el mejoramiento genético del trigo (Triticum aestivum $\mathrm{L}$.) en México, las variedades que los agricultores sembraban eran mezclas de diferentes tipos muy susceptibles a las royas del tallo (Puccinia graminis $f$. sp. tritici) y de la hoja (Puccinia recondita $f$. $s p$. tritici). El mejoramiento genético se inició formalmente en 1945 y para 1948 los agricultores ya disponían de variedades mejoradas, tales como Gabo 48 y Supremo 211, las cuales fueron introducidas de otros países (HERNÁNDEZ, 1984). A partir de 1951, se comenzaron a usar en la producción comercial las variedades Kentana 48, Yaqui 48 y Mayo 48, que resultaron más rendidoras y resistentes a las royas (STAKMAN et al., 1969). A partir de entonces el programa de mejoramiento genético ha desarrollado y liberado un gran número de variedades.

El progreso logrado en el mejoramiento genético del trigo durante la década 1950-1960, fue factor muy importante en la producción, debido a que las variedades mejoradas resultaron más eficientes para producir grano. Sin embargo, el potencial de rendimiento de las variedades de esa década (Lerma Rojo, Huamantla Rojo y Nainari 60) no se podía explotar totalmente, debido a que se encamaban con aplicaciones mayores de $60 \mathrm{~kg} \mathrm{ha}^{-1}$ de nitrógeno. Esa limitación, al aumento del rendimiento impuesta por el encamado, fue la de más peso en la decisión de obtener variedades semi-enanas (Borlaug, 1969).

Hasta 1960, el incremento en el rendimiento promedio nacional fue lento; sin embargo, con la obtención y uso de variedades semi-enanas tales como Pitic S62, Pénjamo 62, Lerma Rojo 64, Mayo 64 y Sonora 64, la aplicación de cantidades mayores de fertilizantes y el uso de mejores prácticas de cultivo, la producción total aumentó al grado en que el país empezó a ser autosuficiente en la producción de este cereal (HewITT, 1982). Durante las últimas cuatro décadas, el rendimiento promedio nacional de trigo en México, se incrementó de $772 \mathrm{~kg} \mathrm{ha}^{-1}$ en 1940 , hasta $4357 \mathrm{~kg} \mathrm{ha}^{-1}$ en 1984 . Este incremento se atribuye al mejoramiento genético y al uso adecuado de mejores prácticas de cultivo. Según JENSEN (1978), en los 40 años de investigación sobre trigo en el estado de Nueva York (1936-1975), el rendimiento se incrementó $100 \%$, pero solamente $49 \%$ de ese total es acreditable al mejoramiento genético. De igual manera, FrEY (1981) señala que el potencial genético de rendimiento de las variedades actuales de trigo de los Estados Unidos, es $50 \%$ superior al de las usadas a principios de siglo. En el Reino Unido, el progreso obtenido con trigo en los últimos 30 años ha sido de $2800 \mathrm{kgha}{ }^{\prime}$ a principios de 1950 , hasta $6160 \mathrm{~kg} \mathrm{ha}^{-1}$ en 1982. De este avance total, solamente el
$50 \%$ es atribuible al mejoramiento genético (AUSTIN et al., 1980 ; SilveY, 1981 y Bingham, 1983).

Al comparar el rendimiento de un grupo de variedades mexicanas, liberadas entre 1962 y 1975 en una región libre de royas (Ciudad Delicias, Chihuahua), se encontró (INIA, 1976) que la variedad más reciente (Anáhuac F75) fue $30 \%$ superior en rendimiento a la variedad liberada en 1962 (Pénjamo T62). Evans (1980) señala que en el programa de mejoramiento de trigo de México, se ha logrado una ganancia total de $5.2 \%$ por año, de la cual solamente $0.9 \%$ por año es atribuible al mejoramiento genético.

Dado que en las estimaciones sobre el avance logrado en el mejoramiento genético del trigo en México, no se han incluido todas las épocas ni las principales variedades liberadas por los programas de mejoramiento genético, el objetivo de este estudio fue examinar, bajo condiciones controladas de plagas, royas y encamado, el avance genético logrado en el rendimiento con las variedades liberadas desde 1950 hasta 1982 y la relación que este carácter guarda con otros caracteres de planta.

\section{MATERIALES Y METODOS}

Los materiales usados en este estudio fueron 81 variedades de trigo que representan las liberadas durante el período 1950-82. La evaluación se hizo durante el ciclo invierno-primavera en 1982-83 y 1983-84 en Roque, Guanajuato (Ambientes 1 y 2) e invierno-primavera 1983-84 y 1984-85 en Chapingo, México (Ambientes 3 y 4). El diseño experimental usado en cada caso, fue un látice simple con dos repeticiones.

Las fechas de siembra fueron 13 y 19 de diciembre en Roque, y 2 y 10 de enero en Chapingo. En todos los casos se usó una densidad de siembra de $120 \mathrm{~kg} \mathrm{ha}^{-1} \mathrm{y}$ una fertilización por hectárea de 100-40-0 al momento de la siembra y 60-0-0 poco antes del amacollo.

La parcela experimental, fue de 4 surcos de $5 \mathrm{~m}$ de largo y $0.30 \mathrm{~m}$ entre surcos en Chapingo y de 6 surcos de $5 \mathrm{~m}$ de largo y $0.15 \mathrm{~m}$ entre surcos en Roque. En Chapingo se cosecharon los dos surcos centrales y en Roque 6 surcos de $3 \mathrm{~m}$ (en esta localidad el rendimiento se ajustó a $3.0 \mathrm{~m}^{2}$ ).

Las plagas y enfermedades se controlaron durante todo el ciclo de cultivo y a las variedades de porte alto se les colocó una espaldera para evitar su encamado. Los cuatro experimentos fueron conducidos totalmente bajo riego, hasta que todas las variedades alcanzaron madurez fisiológica.

Los caracteres medidos o calculados para cada parcela fueron los siguientes : antesis, madurez fisiológica, altura de planta, período de llenado del grano (días de la antesis a la madurez fisiológica), rendimiento 
biológico (rendimiento de paja + grano) (este dato no se determinó en el ambiente 1), rendimiento de grano, índice de cosecha (rendimiento de grano entre rendimiento biológico) (este dato no se determinó en el ambiente 1), rendimiento de grano por día (rendimiento de grano entre días a madurez fisiológica) y tasa de llenado del grano (rendimiento de grano entre período de llenado del grano).

Con los datos obtenidos en cada carácter, se hizo un análisis de varianza para cada ambiente considerando todos los efectos como aleatorios. Dado que las varianzas de los experimentos resultaron heterogéneas, las medias generales de variedades fueron ponderadas como sigue :

$$
\overline{\mathrm{X}}=\frac{\overline{\mathrm{X}} \mathrm{i}_{1} / \mathrm{S}_{1}^{2}+\overline{\mathrm{X}}_{2} / \mathrm{S}_{2}^{2}+\overline{\mathrm{X}} \mathrm{i}_{3} / \mathrm{S}_{3}^{2}+\overline{\mathrm{X}}_{4} / \mathrm{S}_{4}^{2}}{1 / \mathrm{S}_{1}^{2}+1 / \mathrm{S}_{2}^{2}+1 / \mathrm{S}_{3}^{2}+1 / \mathrm{S}_{4}^{2}}
$$

Las medias así obtenidas se agruparon por período de liberación y los promedios de éstos se compararon mediante contrastes, para lo cual se utilizó la varianza y grados de libertad ponderados (MARTínez, 1985) correspondientes a cada carácter.

$$
\begin{gathered}
\hat{\mathrm{S}}^{2}=\frac{1}{1 / \mathrm{S}_{1}^{2}+1 / \mathrm{S}_{2}^{2}+1 / \mathrm{S}_{3}^{2}+1 / \mathrm{S}_{4}^{2}} \\
\mathrm{gl}=\frac{\mathrm{S}_{1}^{2} / \eta_{1}+\mathrm{S}_{2}^{2} / \eta_{2}+\mathrm{S}_{3}^{2} / \eta_{3}+\mathrm{S}_{4}^{2} / \eta_{4}}{\left(\mathrm{~S}_{1}^{2} / \eta_{1}\right)^{2} / \eta_{1}-1+\left(\mathrm{S}_{2}^{2} / \eta_{2}\right)^{2} / \eta_{2}-1} \\
+\left(\mathrm{S}_{3}^{2} / \eta_{3}\right)^{2} / \eta_{3}-1+\left(\mathrm{S}_{4}^{2} / \eta_{4}\right)^{2} / \eta_{4}-1
\end{gathered}
$$

Donde $\mathrm{Xi}=$ media ponderada de la variedad $\langle\mathrm{i}\rangle$ $(i=1 \ldots 32), X i j=$ media de la variedad $\langle i »$ en el ambiente $\left\langle\mathrm{j} »(\mathrm{j}=1 \ldots 4), \mathrm{S}_{\mathrm{j}}{ }^{2}=\right.$ varianza del ambiente " $\mathrm{j} », \hat{\mathrm{S}}^{2}=$ varianza ponderada, $\mathrm{gl}=$ grados de libertad ponderados y $n_{j}=$ grados de libertad del error en el ambiente $\langle j\rangle$.

En la ponderación de las medias de rendimiento, varianzas y grados de libertad, de los ambientes 1,3 y 4 en que hubo ajuste por diseño, se usó el cuadrado medio del error intrabloques y en el ambiente 2 , en que no hubo tal ajuste, se usó el cuadrado medio del error total con sus grados de libertad correspondientes. Este criterio se aplicó para los demás caracteres.

La comparación entre medias de grupos de variedades se hizo mediante contrastes, debido a que se manejó diferente número de variedades por período de liberación. Así, el primer contraste consistió en comparar la media de la variedad Supremo 211 contra la de las variedades del período 1951-55; el segundo contraste, consistió en comparar la media de variedades del período 1951-55 contra la de variedades del período 1956-60 y así sucesivamente.
La estimación del avance genético se hizo con base en las variedades más representativas de cada período ( 32 de las 81 variedades evaluadas), tomando como base a Supremo 211, variedad que se introdujo a México a mediados de la década de 1940-50 y se sembró comercialmente desde 1948 hasta 1951, año en que sucumbió al ataque de las royas (STAKMAN et al., 1969).

Los coeficientes de correlación del rendimiento de grano con otros caracteres de planta, se estimaron con las medias de las 32 variedades, $\mathrm{y}$ el avance en $\mathrm{kg} \mathrm{ha}^{-1}$ año ${ }^{-1}$ obtenido con las variedades nuevas se estimó con base en la regresión de la media del rendimiento de cada periodo sobre la época de liberación de variedades.

\section{RESULTADOS Y DISCUSION}

Además de que la comparación de las variedades se llevó a cabo bajo condiciones favorables de riego, fertilización y control de plagas y enfermedades, las condiciones climatológicas fueron apropiadas para un desarrollo normal del cultivo.

El análisis de varianza combinado con los cuatro ambientes mostró una interacción genotipo-ambiente significativa $(\alpha \leqslant 0.0001)$, lo que indica que las variedades tuvieron un comportamiento diferente a través de ambientes.

El conjuntar datos de un ambiente con condiciones favorables como lo es Roque, Guanajuato, con los de un ambiente con condiciones menos favorables, como lo es Chapingo, México, es muy posible que se esté subestimando el avance genético logrado por el programa de mejoramiento genético del trigo en México. Roque está ubicado en la región del Bajio, donde se cultivan aproximadamente 160000 ha de riego durante el ciclo de invierno-primavera con un rendimiento promedio de $4500 \mathrm{~kg} \mathrm{ha}^{-1}$ y Chapingo se localiza en la región de los Valles Altos de la Mesa Central, donde se cultivan aproximadamente $40 \quad 000$ ha de secano durante el ciclo de verano-otoño, con un rendimiento promedio de $1800 \mathrm{~kg} \mathrm{ha}^{-1}$.

A pesar de las limitaciones señaladas, se hace una evaluación de los resultados obtenidos.

El rendimiento promedio de las variedades liberadas durante los períodos 1951-55 y 1956-60, es superior al de la variedad Supremo 211 en 5 y $31 \%$, respectivamente; con lo que en promedio, las variedades obtenidas durante el periodo $1956-60$ son $26 \%$ superiores a las de 1951-55 (tabla 1). El avance relativamente bajo logrado con las variedades liberadas durante el período 1951-55 puede atribuirse, por un lado, al poco conocimiento sobre el manejo del cultivo que se tenía y por otro, a la presión por generar variedades que superaran, aunque fuera en mínima proporción, a las variedades introducidas o criollas que se sembraban, para justificar con ello la existencia del programa de mejoramiento genético, mientras se seguía trabajando en la obtención de variedades con mayor potencial de rendimiento. Fue así que, para el siguiente período de liberación (195660 ), se obtuvieron las variedades Lerma Rojo, Huaman- 
TABLA 1

Rendimiento de grano promedio y porcentual de variedades liberadas en diferentes periodos. (4 ensayos realizados en Roque, Guanajuato y Chapingo, México.)

Mean grain yield and percent of varieties released in different periods. (4 yield trials conducted in Roque, Gto, and Chapingo, Mex.)

Rendement moyen de grain et gain en pour cent des variétés obtenues à différences périodes (quatre expériences réalisées à Roque, Gto et Chapingo, Mex.).

\begin{tabular}{|c|c|c|c|}
\hline Variedad & $\begin{array}{l}\text { Periodo de } \\
\text { liberacion }\end{array}$ & $\begin{array}{l}\text { Rendimiento } \\
\text { g parcela }^{-1}\end{array}$ & $\%$ \\
\hline Supremo 211 & 1950 & $920 \mathrm{a}$ & 100 \\
\hline Chapala & $1951-55$ & 636 & \\
\hline Yaqui 48 & & 971 & \\
\hline Kentana 48 & & 1,136 & \\
\hline Chapingo 48 & & 861 & \\
\hline Huamantla & & 1,238 & \\
\hline Promedio & & $968 \mathrm{a}$ & 105 \\
\hline Gabo & $1956-60$ & 1,235 & \\
\hline Lerma Rojo & & 1,158 & \\
\hline Huamantla Rojo & & 1,204 & \\
\hline Nainari 60 & & 1,236 & \\
\hline Promedio & & $1,208 \mathrm{~b}$ & 131 \\
\hline Mayo 64 & $1961-65$ & 1,277 & \\
\hline Pitic 62 & & 1,268 & \\
\hline Lerma Rojo 64 & & 1,387 & \\
\hline Pénjamo 62 & & 1,567 & \\
\hline Promedio & & $1,374 \mathrm{c}$ & 149 \\
\hline Tobari 66 & $1966-70$ & 1,261 & \\
\hline Yécora 70 & & 1,437 & \\
\hline INIA 66 & & 1,267 & \\
\hline Siete Cerros 66 & & 1,429 & \\
\hline Super X 66 & & 1,388 & \\
\hline Promedio & & $1,356 \mathrm{c}$ & 147 \\
\hline Anáhuac 75 & $1971-75$ & 1,437 & \\
\hline Toluca 73 & & 1,427 & \\
\hline Salamanca 75 & & 1,608 & \\
\hline Cajeme 71 & & 1,499 & \\
\hline Cleopatra & & 1,405 & \\
\hline Promedio & & $1,475 \mathrm{~d}$ & 160 \\
\hline Hermosillo 77 & $1976-80$ & 1,426 & \\
\hline Jahuara 77 & & 1,457 & \\
\hline Nacozari 76 & & 1,334 & \\
\hline Pavón 76 & & 1,570 & \\
\hline Tesia 79 & & 1,560 & \\
\hline Promedio & & $1,469 \mathrm{~d}$ & 160 \\
\hline Mixteco 82 & $1981-82$ & 1,644 & \\
\hline Abasolo 81 & & 1,527 & \\
\hline Genaro 81 & & 1,629 & \\
\hline Promedio & & $1,600 \mathrm{e}$ & 174 \\
\hline
\end{tabular}

Los promedios con la misma letra son estadisticamente iguales con un nivel de significancia $\alpha=0,05$.

tla Rojo, Nainari 60 y Gabo 60, más rendidoras que sus antecesoras. Lerma Rojo, además de tener mayor potencial de rendimiento, también resultó ser resistente al desgrane y a las royas, lo cual la convirtió en la variedad de trigo más popular en el país, a tal grado que llegó a cubrir más de $60 \%$ de la superficie sembrada con trigo en el país . (O.E.E. 1958).

Con la obtención de variedades semi-enanas, como Pénjamo 62 y Pitic 62, se inició una nueva era en el mejoramiento genético del trigo. Estas variedades, además de soportar sin encamarse mayores cantidades de fertilizantes, también resultaron más resistentes a las royas y más productivas que las variedades de porte alto, debido a que tienen un mayor número de espigas por unidad de superficie y más granos por espiga (datos no presentados).
Aun cuando las variedades liberadas durante los períodos 1966-70 y 1976-80 mostraron un estancamiento en el rendimiento con relación a las variedades del período anterior, el avance en el rendimiento logrado por el programa de trigo de México, vía mejoramiento genético, ha sido lineal y ascendente; tendencia que se observa más claramente en el rendimiento de trigo obtenido a nivel comercial en México (tabla 2).

\section{TABLA 2}

Rendimiento de trigo más bajo, más alto y el promedio obtenido en México en cada periodo de cinco años de 1951 a 1985.

Range of wheat yields and their average obtained in Mexico for each period of five years from 1951 to 1985.

Rendements de blé : minimun, maximun et moyen obtenus au Mexique pour chaque période de cinq ans de 1951-1985.

\begin{tabular}{lccc}
\hline Periodo & $\begin{array}{c}\text { Rendimiento } \\
\text { más bajo }\end{array}$ & $\begin{array}{c}\text { Rendimiento } \\
\text { más alto }\end{array}$ & $\begin{array}{c}\text { Rendimiento } \\
\text { promedio }\end{array}$ \\
\hline & & $\mathrm{kg} \mathrm{ha}^{-1}$ & \\
$1951-55$ & 877 & 1098 & 948 \\
$1956-60$ & 1326 & 1592 & 1457 \\
$1961-65$ & 1676 & 2692 & 2180 \\
$1966-70$ & 2254 & 3020 & 2680 \\
$1971-75$ & 2634 & 3602 & 3216 \\
$1976-80$ & 3464 & 3914 & 3730 \\
$1981-85$ & 3704 & 4409 & 4158 \\
& & & \\
\hline
\end{tabular}

Adaptado de Econotecnia Agrícola No. 9 SARH-DGEA y Dirección General de Desarrollo Agrícola, SARH.

Al comparar el rendimiento de Supremo 211 con el de las variedades del período 1981-82 (Genaro 81, Abasolo 81 y Mixteco 82), se encontró que éstas son superiores a aquélla en $74 \%$. La variedad Mixteco 82 , seleccionada para condiciones de secano, resultó muy productiva bajo condiciones de riego.

El avance significativo logrado con las variedades liberadas en el último período (1981-82), puede atribuirse por un lado, a la combinación de trigos de primavera con trigos de invierno y por otro, a la regionalización ecológica que el Instituto Nacional de Investigaciones Agrícolas empezó a hacer a partir de 1977 en sus programas de mejoramiento genético; regionalización que ha permitido aprovechar la interacción genotipo ambiente al obtener variedades apropiadas para condiciones ambientales más específicas.

Si se considera que la investigación en trigo en México se inició formalmente en 1945, el avance genético total obtenido en el rendimiento de grano representa una tasa de incremento anual de $2.0 \%$ o alrededor de $60 \mathrm{~kg} \mathrm{ha}^{1}$ año '. Esta tasa de incremento en el rendimiento es mayor a la obtenida en programas de mejoramiento genético de otros países (JENSEN, 1978 ; EvANS, 1980 ; FREY, 1981 y Bingham, 1983), y a la obtenida por WADDINGTON et al., 1986 para la principal región triguera de México.

Es de hacer notar que las variedades Huamantla Rojo, Pénjamo 62, Yécora 70, Cleopatra 74, Salamanca 75, Tesia 79 y Genaro 81 , tendieron a tener consistentemente el mayor rendimiento de grano dentro de su grupo a través de años y localidades. Estos resultados indican que la selección y liberación de variedades por el 
TABLA 3

Medias de caracteres de variedades liberadas en diferentes periodos y el nivel de significación ( $\alpha$ ) entre paréntesis del contraste correspondiente. Mean traits of varieties released in different periods and the level of significance $(\alpha)$ in parentheses of the corresponding contrast.

Corrélation du rendement en grain avec d'autres caractères de la plante et son niveau de signification ( $\alpha)$ entre parenthèses.

\begin{tabular}{|c|c|c|c|c|c|c|c|c|c|}
\hline $\begin{array}{l}\text { Período de } \\
\text { Liberación }\end{array}$ & $\begin{array}{l}\text { Número de } \\
\text { variedades }\end{array}$ & $\begin{array}{c}\text { Rendimiento } \\
\text { de grano } \\
\text { (g/parcela) }\end{array}$ & $\begin{array}{l}\text { Rendimiento } \\
\text { por día } \\
\text { (g/parcela/día) }\end{array}$ & $\begin{array}{c}\text { Rendimiento } \\
\text { biológico } \\
\text { (g/parcela) }\end{array}$ & $\begin{array}{l}\text { Indice de } \\
\text { cosecha (1) }\end{array}$ & $\begin{array}{l}\text { Tasa llenado } \\
\text { de grano } \\
\text { (g/parcela/día) }\end{array}$ & $\begin{array}{l}\text { Antesis } \\
\text { (días) }\end{array}$ & $\begin{array}{l}\text { Madurez } \\
\text { fisiológica } \\
\text { (días) }\end{array}$ & $\begin{array}{l}\text { Altura } \\
(\mathrm{cm})\end{array}$ \\
\hline 1950 & 1 & $\begin{array}{c}920 \\
(0.4216)\end{array}$ & $\begin{array}{c}6.70 \\
(0.1205)\end{array}$ & $\begin{array}{c}4722 \\
(0.2808)\end{array}$ & $\begin{array}{c}0.170 \\
(0.0000)\end{array}$ & $\begin{array}{c}27.60 \\
(0.0177)\end{array}$ & $\begin{array}{c}105 \\
(0.0000)\end{array}$ & $\begin{array}{c}139 \\
(0.0000)\end{array}$ & $\begin{array}{c}129 \\
(0.0175)\end{array}$ \\
\hline $1951-55$ & 5 & $\begin{array}{c}968 \\
(0.0000)\end{array}$ & $\begin{array}{c}7.42 \\
(0.0000)\end{array}$ & $\begin{array}{c}4506 \\
(0.0244)\end{array}$ & $\begin{array}{c}0.232 \\
(0.2500)\end{array}$ & $\begin{array}{c}23.96 \\
(0.0000)\end{array}$ & $\begin{array}{c}91 \\
(0.0000)\end{array}$ & $\begin{array}{c}132 \\
(0.0062)\end{array}$ & $\begin{array}{c}124 \\
(0.0000)\end{array}$ \\
\hline $1956-60$ & 4 & $\begin{array}{c}1208 \\
(0.0000)\end{array}$ & $\begin{array}{c}9.12 \\
(0.0000)\end{array}$ & $\begin{array}{c}4784 \\
(0.0804)\end{array}$ & $\begin{array}{c}0.227 \\
(0.0000)\end{array}$ & $\begin{array}{c}29.45 \\
(0.0006)\end{array}$ & $\begin{array}{c}93 \\
(0.0006)\end{array}$ & $\begin{array}{c}133 \\
(0.3459)\end{array}$ & $\begin{array}{c}119 \\
(0.0000)\end{array}$ \\
\hline 1961-65 & 4 & $\begin{array}{c}1374 \\
(0.6186)\end{array}$ & $\begin{array}{c}10.42 \\
(0.4271)\end{array}$ & $\begin{array}{c}4557 \\
(0.8185)\end{array}$ & $\begin{array}{c}0.278 \\
(0.2877)\end{array}$ & $\begin{array}{c}32.87 \\
(0.0584)\end{array}$ & $\begin{array}{c}91 \\
(0.0083)\end{array}$ & $\begin{array}{c}132 \\
(0.8421)\end{array}$ & $\begin{array}{c}100 \\
(0.0000)\end{array}$ \\
\hline $1966-70$ & 5 & $\begin{array}{c}1356 \\
(0.0007)\end{array}$ & $\begin{array}{c}10.20 \\
(0.0000)\end{array}$ & $\begin{array}{c}4529 \\
(0.2598)\end{array}$ & $\begin{array}{c}0.270 \\
(0.0000)\end{array}$ & $\begin{array}{c}31.10 \\
(0.0000)\end{array}$ & $\begin{array}{c}90 \\
(0.0000)\end{array}$ & $\begin{array}{c}132 \\
(0.0000)\end{array}$ & $\begin{array}{c}89 \\
(0.0394)\end{array}$ \\
\hline $1971-75$ & 5 & $\begin{array}{c}1475 \\
(0.8674)\end{array}$ & $\begin{array}{c}11.40 \\
(0.2314)\end{array}$ & $\begin{array}{c}4399 \\
(0.0002)\end{array}$ & $\begin{array}{c}0.303 \\
(0.0012)\end{array}$ & $\begin{array}{c}34,50 \\
(0.7854)\end{array}$ & $\begin{array}{c}88 \\
(0.0000)\end{array}$ & $\begin{array}{c}130 \\
(0.0000)\end{array}$ & $\begin{array}{c}87 \\
(0.0176)\end{array}$ \\
\hline $1976-80$ & 5 & $\begin{array}{c}1469 \\
(0.0013)\end{array}$ & $\begin{array}{c}11.08 \\
(0.1424)\end{array}$ & $\begin{array}{c}4845 \\
(0.2808)\end{array}$ & $\begin{array}{c}0.280 \\
(0.0274)\end{array}$ & $\begin{array}{c}35,36 \\
(0.5513)\end{array}$ & $\begin{array}{c}93 \\
(0.0000)\end{array}$ & $\begin{array}{c}133 \\
(0.1818)\end{array}$ & $\begin{array}{c}90 \\
(0.0027)\end{array}$ \\
\hline 1981-82 & 3 & 1600 & 11.53 & 4807 & 0.298 & 35.96 & 91 & 133 & 94 \\
\hline
\end{tabular}

(1) El indice de cosecha está calculado con las medias de los ambientes 2. 3, 4.

progama de mejoramiento genético de trigo de México ha sido acertada, ya que son las variedades más productivas y que más se han sembrado comercialmente. Algunas de las variedades liberadas hace más de 10 años, aún se usan con gran aceptación, como es el caso de Salamanca 75 en la región del Bajío y Cleopatra 74 en la región de los Valles Altos de la Mesa Central.

Dado que en México el mejoramiento genético del trigo se ha enfocado a generar variedades resistentes a las royas que aparecen contínuamente, al sustituir variedades que paulatinamente se tornan susceptibles por otras más resistentes, se ha descuidado la selección de variedades con mayor potencial de rendimiento. Esta podría ser una explicación al estancamiento que se tiene en el rendimiento de las variedades liberadas durante los períodos 1966-70 y 1976-80, donde se observa una reducción en el índice de cosecha y en el rendimiento de grano por dia que se presenta en las variedades de dichos períodos, con relación a las del período anterior. Es sabido que la tendencia del programa de mejoramiento genético del trigo en México, ha estado orientada a producir variedades altamente resistentes y de amplia adaptación, a expensas del rendimiento de grano, lo cual ha sido el motivo de no tener mayores avances.

La comparación entre las medias del rendimiento de grano con las de otros caracteres de planta (tabla 3) y los análisis de correlación (tabla 4), muestran que el rendimiento de grano está relacionado positivamente con rendimiento de grano por día, índice de cosecha, tasa y período de llenado del grano, y negativamente con la altura de la planta. El rendimiento biológico y la madurez fisiológica no presentan asociación alguna con el rendimiento de grano. Se observa que el indice de
TABLA 4

Correlaciones de Rendimiento de Grano con otros Caracteres de Planta $y$ su nivel de significancia $(\alpha)$ entre paréntesis.

Correlations of grain yield with other plant traits and the level of significance in parentheses.

Moyennes des caractères des variètés obtenues à différentes périodes et le niveau de signification $(\alpha)$, entre parenthèses leurs contrastes.

\begin{tabular}{lr}
\hline \hline Caracter & Correlacion \\
\hline Rendimiento Biológico & 0.31524 \\
& $(0.0788)$ \\
Indice de Cosecha & 0.68512 \\
& $(0.0001)$ \\
Rendimiento de grano/dia & 0.98345 \\
& $(0.0001)$ \\
Tasa de llenado de grano & 0.93045 \\
& $(0.0001)$ \\
Periodo de llenado de grano & 0.65511 \\
& $(0.0001)$ \\
Altura de Planta & 0.75363 \\
& $(0.0001)$ \\
Madurez Fisiológica & 0.00264 \\
& $(0.9886)$ \\
\hline
\end{tabular}

cosecha de las variedades liberadas hasta 1975 se incrementó paralelamente con el rendimiento de grano, sin embargo, el índice de cosecha y la altura de planta de las variedades liberadas de 1976-82 cambiaron su tendencia. Esto podría indicar que el índice de cosecha de las variedades mexicanas de trigo alcanzó un óptimo como ha sido el caso en las variedades de avena (LAwES, 1977).

El mayor rendimiento de grano que las variedades más recientes manifiestan en comparación con sus antecesoras y la obtención de líneas experimentales con mayor potencial de rendimiento a partir de dichas 
variedades, por el programa de mejoramiento genético de trigo de México, mantienen viva la posibilidad de seguir aumentando el rendimiento de grano en dicho programa.

\section{CONCLUSIONES}

El mayor rendimiento de grano de las variedades nuevas de trigo sobre las más antiguas, puede atribuirse a la mayor resistencia al encamado, mayor índice de cosecha y mayor tasa y período de llenado del grano que caracteriza a las variedades nuevas.

Con pequeñas variaciones, durante los períodos de 1966-70 y 1976-80, el avance en rendimiento de grano logrado en los 37 años de investigación en trigo (19451982) ha sido lineal y ascendente, con un avance total de $74 \%$, el cual representa una tasa de incremento anual de $2 \%$ ó alrededor de $60 \mathrm{~kg} \mathrm{ha}^{1}$ año ${ }^{1}$. Los resultados obtenidos de este estudio, aunados con la obtención de genotipos con mayor potencial de rendimiento que se tienen en el programa de mejoramiento genético, permiten anticipar que se podrá continuar incrementando el rendimiento de grano y mantener la resistencia a las royas, así como la amplia adaptación de las variedades mexicanas de trigo.

Recu le 20 janvier 1987. Accepté le 10 mai 1988.

\section{RECONOCIMIENTO}

Agradesco a los ings. Ricardo Urbina A. y Salomon solano H. investigadores del INIFAP su valiosa ayuda en los trabaros de campo. Se agradece la valiosa revisión que hicieron a este artículo los doctores Alfredo Carballo Quirós, Profesor-Investigador del Centro de Genética del Colegio de Postgraduados y Dana Eaton, Investigador del Programa de Maiz del Centro Internacional de Mejoramiento de Maíz y Trigo. Al Dr. Angel Martínez GarzA, Profesor-Investigador del Centro de Estadistica y Cálculo del Colegio de Postgraduados, y al M. C. Gerardo Terrazas estadistico del CIFAP-MEX se agradece su ayuda en el análisis estadístico.

\section{LITERATURA CITADA}

Austin R. B., Bingham J., Blackwell R. D., Evans L. T., Ford M. F., Morgan C. C., Taylor M., 1980. Genetic improvements in winter wheat yields since 1900 and associated physiological changes. J. Agric. Sci., Cambridge, 94 : 675-689.

Bingham J., 1983. Genetic Constraints on Progress in Wheat Breeding. Plant Breeding Institute, Trumpington, Cambridge, England, Pamphlet, 9 p.

Borlaug N. E., 1969. Mejoramiento del Trigo: Su impacto en el abastecimiento mundial de alimentos. Sobretiro, el Batán, México, CIMMYT, n², 1-40.

Evans L. T., 1980. The natural history of crop yield. Am. Sci., 68 : 388-397.

Frey K. J., 1981. Capabilities and limitations of conventional plant breeding. In Genetic Engineering for crop improvement. A Rockefctler Foundation Conference. The Rockefeller Foundation, New York, N.Y. 15-62.

Hernández S. A., 1984. Antecedentes del mejoramiento genético del trigo en México. En : Germen, Bol. Intercambio Cientifico SOMEFI$M E X I C O . \mathrm{n}^{\circ} 2,32-45$.

Hewitt de Alcantara C., 1982. La modernización de la agricultura mexicana 1940-1970. Ed. Siglo Veintiuno, 3a. Edición. México. 31-56.
INIA ; 1976. Resultatdos en Foques y proyección de la investigación en el cultivo del trigo en México. En : INIA $X V$ años de investigación agricola. INIA SAG, Mexico. 303-312.

Jensen N. F., 1978. Limits to growth in world food production. Science, $201: 317-320$.

Lawes D. A., 1977. Yield improvement in spring oats. J. Agric. Sci., $89: 751-757$.

Martínez G. V., 1985. Diseños Experimentales, Colegio de Postgraduados, Chapingo, México. CIMMYT, 967-1012.

OEE ; 1958. Mejoramiento del trigo. En : Adelantos en la investigatión. Informe anual 1957-58. Oficina de estudios especiales, $S A G$ México. 85-127.

Stakman E. C., Bradfield R., Mangelsdorf P. C., 1969. Campañas contra el hambre. Traducido por Rafael Castillo Dibildox. Ed. Uteha (Unión Tipográfica Editorial Hispano-americana). México.

Silvey V., 1981. The contribution of new wheat, barley and oat varieties to increasing yield in England and Wales 1947-78. J. Nat. Inst. Agr. Bot., 15:399-412.

Waddinton S. R., Ransom J. K., Osmanzai M., Saunders D. A., 1986. Improvement in yield potential of bread wheat adapted to north-west of Mexico. Crop. Sci., 26:698-703. 